94/165 (60\%) respondents had their own children (201 children). Mode of delivery for first born child; normal vaginal $48 \%$, caesarean section $26.5 \%$ (elective $8.5 \%$, emergency $18 \%$ ), instrumental $24.5 \%$ and vaginal breech $1 \%$. Elective caesarean was performed in $8.5 \%$. Only one chose an elective caesarean for maternal request. SW obstetricians statistically have the same overall actual modes of birth (Kruksal-Wallis $\mathrm{p}=0.932$ ).

Conclusions $10 \%$ report they would request caesarean section for themselves/their partner, which is the lowest rate reported within UK studies. Only $1 \%$ had a caesarean solely for maternal choice. SW Obstetricians would choose non interventional delivery if possible. They currently have modes of delivery that are not statistically different from the general population. These results challenge long held misconceptions about birth choices made by obstetricians.

\section{PL.16 DOES THE USE OF CARBETOCIN REDUCE RECOVERY TIMES AT CAESAREAN SECTION? AN AUDIT OF OUTCOMES FOLLOWING ROUTINE INTRODUCTION OF CARBETOCIN AT SOUTHMEAD HOSPITAL}

doi:10.1136/archdischild-2013-303966.200

'EH Shaw, ${ }^{2} \mathrm{E}$ Bækgaard, 'D Siassakos, 'TJ Draycott. 'Southmead Hospital, Bristol, UK; ${ }^{2}$ Bristol University, Bristol, UK

\section{Objectives}

1. To investigate time in theatre recovery for women who received carbetocin at Caesarean Section (CS) compared with a historical cohort.

2. To compare costs per patient, from a health sector perspective, between the two cohorts.

Methods We evaluated outcomes for all women (elective and emergency) undergoing CS, after the introduction of carbetocin in April 2012. The controls comprised every $3^{\text {rd }}$ patient undergoing CS in January 2012 (pre-carbetocin).

Main outcome measure Difference in time in theatre recovery between the two groups.

Results Women who received carbetocin $(n=265)$ spent less time in recovery than the historical cohort $(n=33)$ (carbetocin $170 \mathrm{~min}$, syntocinon $271 \mathrm{~min}$; difference: -101.3023 minutes, 95\% CI: $-175.8518 ;-26.75276, \mathrm{p}<0.01)$.

Additionally there was reduced need for additional $3^{\text {rd }}$ stage uterotonics (carbetocin 16\%, syntocinon 60\%; mean difference in proportion: $-0.294,95 \% \mathrm{CI}:-0.1183$; -0.4697$)$. This is consistent with findings from RCTs.

Using financial modelling (Abstract No: PL.19) drug cost per patient when all $3^{\text {rd }}$ stage requirements are included is carbetocin $£ 7.78 \mathrm{v}$ syntocinon $£ 6.37$. In addition, reduced theatre recovery time has potential midwifery staffing cost efficiencies of up to $£ 189,000 \mathrm{pa}$. Conclusion Carbetocin decreases time spent in recovery post-CS, and reduces the need for additional $3^{\text {rd }}$ stage management.

Discussion Introducing carbetocin routinely for all CS will reduce recovery times and potentially constitutes a cost saving. There are likely to be additional important staffing and theatre efficiencies.

\section{PL.17 TESTING FOR LIKELIHOOD OF PRE-TERM LABOUR - A DESCRIPTIVE STUDY OF ENGLISH MATERNITY UNITS 2011-2012}

doi:10.1136/archdischild-2013-303966.201

CEM Aiken, M Malina, JC Brockelsby. Addenbrooke's Hospital, Cambridge, UK

Introduction Prematurity is a leading cause of neonatal morbidity and mortality. Tests are available to help predict the likelihood of pre-term labour (PTL), although optimal protocols remain uncertain. We assessed the changing pattern of testing in English maternity units.
Methods 163 maternity units were surveyed online in Sept/Oct 2011, and again in Sept/Oct 2012. In 2012, non-responders were followed up by telephone contact. The overall response rate improved from 32.5\% (54 units) in 2011 to 73\% (119 units) in 2012. Data were analysed quantitatively using contingency tables, and spatially using Geomapping software.

Results In 2012, 87\% (CI; 80-92\%) of units used biochemical testing to predict PTL, a significant $(\mathrm{p}<0.05)$ increase from $2011(76 \%$, CI; $63-85 \%$ ). For units where data were available for both years, $33 \%$ altered their method of PTL testing between 2011-2012, with $40 \%$ of these initiating biochemical testing. 14 units did not test for pre-term labour (11\%, CI; 7-18\%). The most commonly cited barriers to testing were cost and inexperience of operators, each cited by $16 \%$ of units (CI; 10-24\%). On the basis of test results, $94 \%$ (C1; 87-97) of units gave steroids, but only 77\% (CI; 67-84) discharged home and $82 \%$ (CI; 73-88\%) arranged in utero transfer.

Conclusions Our results suggest a heterogeneous pattern of test utilisation. The high proportion of units changing methods within a year implies confusion regarding optimal strategies for PTL prediction. There is an urgent need for further research and clearer guidance in this area. Heterogeneity in protocols could lead to suboptimal allocation of valuable neonatal network resources.

\section{PL.18 VALIDATION OF A SKILL LIST OF NON-TECHNICAL SKILLS FOR OBSTETRICIANS WHEN PERFORMING AN OPERATIVE VAGINAL DELIVERY}

doi:10.1136/archdischild-2013-303966.202

${ }^{1} \underline{G}$ Cass, ${ }^{2} \mathrm{~K}$ Goyder, ${ }^{2 B}$ Strachan, ${ }^{2 R}$ Bahl. 'Musgrove Park Hospital, Taunton, UK; ${ }^{2}$ St Michaels Hospital, Bristol, UK

Background Non-technical skills for obstetricians play a vital role in adequate patient care and combine social and cognitive elements that are separate from the practical skills involved in obstetric care. Categories of non-technical skills for operative vaginal delivery have been postulated from healthcare professionals to provide a framework of good practise.

Aim of this study To validate the social non-technical skills defined by obstetricians and midwives.

Method Women who had an operative vaginal delivery of a term baby underwent a semi structured interview 6-8 weeks postnatal. The interview recordings were transcribed verbatim. Thematic coding of data was carried out. Consistency of interpretation was ascertained by two researchers. Data was analysed to compare and contrast the emerging themes to the elements and categories previously identified.

Results 16 interviews were transcribed and analysed for this study. Social categories of non-technical skills, namely professional relationship, behaviour, teamwork and communication, suggested by obstetricians and midwives were common themes from all participants. New themes emerged such as 'importance of understanding preconception of what operative vaginal delivery would involve'.

Conclusion The non-technical skills developed by obstetricians and midwives in operative vaginal delivery have been validated by women. Furthermore new themes emerged that need to be embedded into the pre-existing categories. This validated framework of non technical skills is vital to ensure an operative vaginal delivery occurs in an environment that is positive and respects the unique event of a birth of a child. Additionally the framework can be used as a tool for training and feedback.

\section{PL.19 COST COMPARISON OF ROUTINE CARBETOCIN USE AT CAESAREAN SECTION}

doi:10.1136/archdischild-2013-303966.203

'EH Shaw, ${ }^{2}$ B Bækgaard, 'D Siassakos, 'TJ Draycott. 'Southmead Hospital, Bristol, UK; ${ }^{2}$ Bristol University, Bristol, UK 


\section{Objectives}

(1) To establish a cost consequence evaluation following introduction of routine use of carbetocin for all Caesarean Sections (CS) at Southmead Hospital.

Methods Following routine introduction of carbetocin for all CS (elective and emergency) at Southmead Hospital in April 2012 we have demonstrated a reduction in theatre recovery time and also need for additional $3^{\text {rd }}$ stage management compared with historical data from pre-carbetocin (Abstract No: PL.16).

We have economically modelled the financial cost of introducing carbetocin, using estimated costs as indicated (table 1).

Conclusion Using real life data following routine introduction of carbetocin, economic modelling demonstrates only a small increase in drug cost per patient (carbetocin $£ 7.78$ v syntocinon £6.37). In addition reduced theatre recovery time has potential midwifery staffing cost efficiencies of up to $£ 189,000 \mathrm{pa}$.

\section{PL.20 PERINEAL ASSESSMENT AND REPAIR E-LEARNING SYSTEM (EPEARLS): AN E-LEARNING TRAINING PACKAGE TO IMPROVE CLINICAL MANAGEMENT OF PERINEAL TRAUMA FOLLOWING CHILDBIRTH}

doi:10.1136/archdischild-2013-303966.204

'A Mahmud, ${ }^{2} \mathrm{C}$ Kettle, ${ }^{4} \mathrm{D}$ Bick, ${ }^{2} \mathrm{C}$ Rowley, ${ }^{3} \mathrm{~T}$ Rathod, ${ }^{3} \mathrm{~J}$ Belcher, ${ }^{2} \mathrm{M}$ Abdelmaguid 1.5K Ismail. 'Birmingham Women's Hospital NHS Foundation Trust, Birmingham, UK,

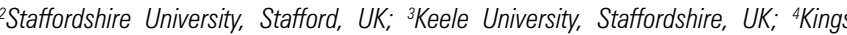
College London, London, UK: ${ }^{5}$ University of Birmingham, Birmingham, UK

Background Birth related perineal trauma can have a major impact on women's health. The correct assessment and repair of perineal injuries are procedures that require knowledge and skill. Currently, there is no agreement as to what constitutes an effective training programme. We produced and tested an interactive distance learning multi-professional training package called ePEARLS. This was developed as a tool for the delivery and assessment of perineal repair training in line with UK evidence based practise.

Methods The main aim of this project was to develop, refine and assess ePEARLS as a tool for the assimilation of knowledge and skill to clinicians involved in Intrapartum care. Using pre and post-training objective assessments for both knowledge and skill, we compared the effect of delivering training using ePEARLS compared to other training models in three independent multi-professional groups from three different maternity units.

Results Thirty-eight participants were involved in the study. Improvements in knowledge scores were marginally significant in all three groups. However, more marked improvements in skill scores were noted in response to the different training models.

Conclusion ePEARLS is the first multi-professional interactive perineal trauma management e-learning package that has been for mally tested against other training models. The potential cost effectiveness of this package makes it far superior to other methods of delivering training and maintaining competency.

\section{PL.21 DELIVERY OUTCOMES FOR NULLIPAROUS WOMEN AT THE EXTREMES OF MATERNAL AGE - A COHORT STUDY}

doi:10.1136/archdischild-2013-303966.205

'DA Vaughan, ${ }^{2,4} \mathrm{~B}$ Cleary, ${ }^{2,3} \mathrm{DJ}$ Murphy. ${ }^{1} T$ The Rotunda Hospital, Dublin, Ireland; ${ }^{2} T$ The Coombe Women and Infants University Hospital, Dublin, Ireland; ${ }^{3}$ Trinity College Dublin, Dublin, Ireland; ${ }^{4}$ The Royal College of Surgeons in Ireland, Dublin, Ireland

Objective To examine the associations between extremes of maternal age ( $\leq 17$ years or $\geq 40$ years) and delivery outcomes.

Design Retrospective cohort study.

Setting Urban maternity hospital in Ireland.

Population A total of 36,916 nulliparous women with singleton pregnancies who delivered between 2000 and 2011.

Methods The study population was subdivided into five maternal age groups based on age at first booking visit: $\leq 17$ years, 18-19 years, $20-34$ years, $35-39$ years and women aged $\geq 40$ years. Logistic regression analyses were performed to examine the associations between extremes of maternal age and delivery outcomes, adjusting for potential confounding factors.

Main Outcome Measures Preterm birth, low birth weight, admission to the neonatal unit, congenital anomaly, caesarean section.

Results Compared to maternal age 20-34 years, age $\leq 17$ years was a risk factor for preterm birth (adjOR 1.83, 95\% CI 1.33-2.52). Babies born to mothers $\geq 40$ years were more likely to require

Abstract PL.19 Table 1

\begin{tabular}{|c|c|c|c|c|c|c|c|}
\hline & & Mistactia & 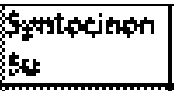 & dentestats & {$[+14$} & tortetenth & 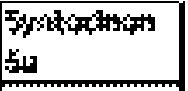 \\
\hline Exfen fosta & & Ents & 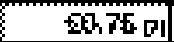 & & ilpth & Fi2ukt & F1,98: \\
\hline 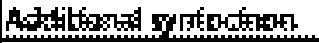 & & 158 & B.40\% & Ex: & & $16 \mathrm{x}$ & ESOS \\
\hline \multirow[t]{3}{*}{ 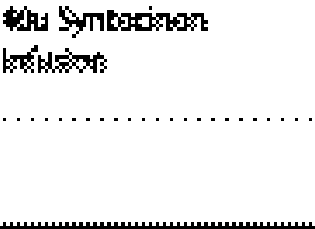 } & & $4,0 \%$ & andsen & & & & \\
\hline & 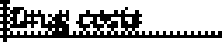 & & & E.9.4. & & 947.6 & 2.41485 \\
\hline & 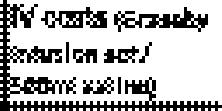 & & & (8) & & 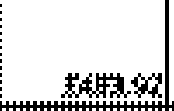 & 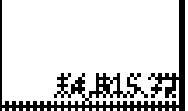 \\
\hline \multirow[t]{2}{*}{ 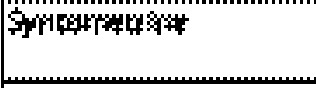 } & & $6+2$ & 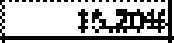 & & & & \\
\hline & 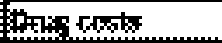 & & & M 10 & & 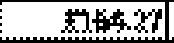 & $\$ \$ 8 \infty$ \\
\hline \multirow[t]{2}{*}{ HaEnghate } & & 1.48 & xㅛㅛ & & & & \\
\hline & 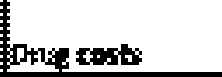 & & & 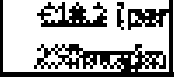 & & 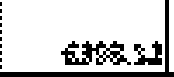 & $+1,2$ \\
\hline 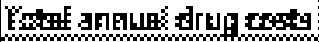 & & & & & & 5140 & $E 1,+7=$ \\
\hline G'tey cost pest & & & & & & 玉7.7. & f6.37] \\
\hline 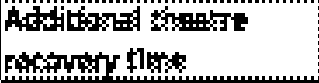 & 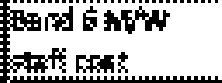 & & Morim & $\forall 8$ & & & $10 \times 0$ \\
\hline
\end{tabular}

1: South West Obstetric Network drug cost; 2: Unit drug cost, 3: NHS supply chain unit cost, 4:PSSRU 2010 Unit Health Care cost 